\title{
The Automated Prediction of Hearing Thresholds in Sleeping Subjects Using Auditory Steady-State Evoked Potentials
}

\author{
Gary Rance, Field W. Rickards, Lawrence T. Cohen, Sandra De Vidi, and \\ Graeme M. Clark
}

\begin{abstract}
Objective: To examine the relationship between auditory steady-state evoked potentials (SSEPs) and behavioral thresholds in sleeping subjects.
\end{abstract}

Design: 60 adults and children with hearing thresholds ranging from normal to profound were selected on the basis of appropriate audiograms. Behavioral audiograms were determined at the octave frequencies 250-4000 Hz. These behavioral thresholds were then compared with the SSEP thresholds obtained during natural sleep for adults, or natural or sedated sleep for children.

Results: A strong relationship between behavioral and SSEP thresholds was observed. The strength of the relationship increased with increasing frequency and increasing degree of the loss. On the basis of these data, the prediction of behavioral thresholds from SSEP levels was determined. It was found that the standard deviation of the error in this prediction decreased with increasing frequency and increasing degree of the loss. There was no significant age effect in the results obtained at any of the frequencies.

Conclusion: The results suggest that the SSEP technique can be used as a predictor of behavioral thresholds in adults and children at the frequencies 250-4000 Hz.

(Ear \& Hearing 1995;16;499-507)

Auditory evoked potentials can offer insight into the hearing acuity of very young and difficult to test subjects. Auditory assessment is especially important in the early detection, diagnosis, and audiological management of hearing impaired children (Markides, 1986; Ramkalawan \& Davis, 1992).

The auditory brain stem response (ABR) is extensively used as an objective method of hearing estimation. There are two features of this technique which have made it particularly suitable for use with subjects unable or unwilling to provide reliable

Human Communication Research Centre (G.R., F.W.R., L.T.C., G.M.C.), Department of Otolaryngology; Deafness Studies Unit (F.W.R, S.D.V.), Department of Learning, Assessment and Special Education, The University of Melbourne, Parkville, Victoria, Australia volitional responses. First, the response is not affected by either sleep or sedation; and second, unlike some of the later auditory potentials, it has been shown to be consistently recordable in subjects of all ages including neonates (Picton, Durieux-Smith, \& Moran, 1994). The successful recording of the ABR is contingent upon the use of a stimulus which has a rapid rise time. $A B R$ responses are most clearly seen when using a wide band click stimulus. However, as the click stimulus produces evoked thresholds typically reflecting the average hearing or best threshold in the region of 1 to $4 \mathrm{kHz}$, it provides limited frequency specific information (Stapells, 1989).

Recent work using ABRs elicited by short duration tones rather than click stimuli, has shown that threshold estimates can be made with reasonable accuracy across a range of stimulus frequencies (Stapells, Picton, Durieux-Smith, Edwards, \& Moran, 1990). However, ABR thresholds are often elevated when subjects with normal behavioral thresholds are used, especially at the lower frequencies (Gorga, Kaminski, Beauchaine, \& Jesteadt, 1988). When using short duration tones, masking noise is required to ensure that the acoustic splatter caused by the rapid onset of the tone does not stimulate regions of the cochlea other than that corresponding to the nominal frequency of the tone (Beattie \& Kennedy, 1992).

An alternative frequency specific approach which does not suffer the spectral distortion problems associated with short duration stimuli is the steadystate evoked potential (SSEP) technique (Kuwada, Batra, \& Maher, 1986; Rickards \& Clark, 1984). SSEPs are scalp potentials elicited in response to a periodically varying stimulus such as a sinusoidal amplitude and/or frequency modulated tone. The resulting potential is periodic and is phase locked to the modulation envelope of the stimulus. Responses can be obtained for a large range of modulation frequencies: 4 to $450 \mathrm{~Hz}$ (Rickards \& Clark, 1984). SSEPs are generally characterized by the amplitude and phase of their first and second harmonics.

Studies have shown that the SSEP response can be automatically detected by real-time statistical 
analysis of samples of the response phase (Jerger, Chimiel, Frost, \& Coker, 1986; Stapells, Makeig, \& Galambos, 1987). Although the " $40 \mathrm{~Hz}$ response" has been used to obtain good estimations of behavioral thresholds in normal and hearing impaired subjects at low and high frequencies (Reference note 1 ), the response is affected by sleep or sedation (Brown \& Shallop, 1982; Galambos, Makieg, \& Talmachoff, 1981; Osterhammel, Shallop, \& Terkildsen, 1985; Shallop, 1983), and response thresholds are elevated and less reliable during sleep (Picton, Vajsar, Rodriguez, \& Campbell, 1987). However, when modulation rates above $70 \mathrm{~Hz}$ are used, responses can be consistently recorded during sleep and at low sound pressure levels (Cohen, Rickards, \& Clark, 1991). Aoyagi et al. (1993) reported reliable responses to sinusoidal amplitude modulated tones in sleeping adults and young children at the modulation frequencies of 80 to $100 \mathrm{~Hz}$. Similarly, Levi, Folsom, and Dobie (1993) found $80 \mathrm{~Hz}$ to be the optimal modulation rate to obtain clear SSEP responses in sleeping adults and infants. Further studies with neonates using these high modulation frequencies have shown that this evoked potential technique is a fast, reliable, and accurate automatic procedure suitable for mass screening (Rickards et al. 1994).

The ability to consistently record SSEPs from sleeping subjects at these higher modulation rates may be due to the source of the response and studies suggest that the latencies of these responses in sleeping subjects are in the order of 10 to $15 \mathrm{msec}$ depending on carrier frequency and modulation rate (Cohen et al., 1991). Further, higher modulation rates can produce latencies of less than 10 msec (Kuwada et al., 1986; Rickards \& Clark, 1984). Latencies of about $10 \mathrm{msec}$ are considered to be most effective when using SSEPs to assess the hearing of sleeping infants and young children (Cohen et al., 1991). Evoked potentials with latencies around $10 \mathrm{msec}$ have been consistently recorded in sleeping newborns and infants (Hawes \& Greenberg, 1981; Shallop \& Osterhammel, 1983). The use of SSEPs in the assessment of hearing in infants and children may also require latencies of about $10 \mathrm{msec}$, and to achieve this, modulation frequencies greater than $70 \mathrm{~Hz}$ may be the most suitable.

To evaluate the applicability of SSEPs for threshold testing in sleeping subjects, this study had two main aims: first, to examine the relationship between SSEP and behavioral thresholds, and second, to use this relationship to determine the accuracy with which SSEP thresholds could predict behavioral thresholds.

\section{MeThod}

\section{Subjects}

There were 60 subjects in this study. Twenty-five subjects were moderately to profoundly hearing impaired children aged 10 to $58 \mathrm{mo}$ (mean age $=29 \mathrm{mo}$; median age $=28 \mathrm{mo}$ ). Thirty-five subjects were adults aged 24 to $82 \mathrm{yr}$ (mean age $=55.7 \mathrm{yr}$; median age $=57 \mathrm{yr}$ ) with audiometric thresholds ranging from normal to profound. The child subjects were selected from patients who were seen for audiological assessment at the Royal Victorian Eye and Ear Hospital. The adult subjects were selected from patients seen by the School of Audiology at The University of Melbourne. The subjects were selected on the basis of appropriate audiograms, and consent to further testing.

\section{Apparatus and Procedures}

Behavioral audiograms were established for all subjects under a standard threshold search procedure using a clinical audiometer (Madsen OB 822) to generate pure or warble tones at octave frequencies from $250 \mathrm{~Hz}$ to $4000 \mathrm{~Hz}$. Results were typically obtained under headphones; however, three of the younger children were assessed in the free field. The hearing losses were all sensorineural in nature with no subject showing any evidence of middle ear pathology or retrocochlear dysfunction.

At the time of the SSEP data collection, all of the adult subjects were in natural sleep. The children were in natural sleep $(n=5)$, sedated with chloral hydrate $(50 \mathrm{mg} / \mathrm{kg}),(n=10)$, or under a general anesthetic (halothane 1:1 nitrous oxide) $(n=10)$.

EEG activity was recorded using silver-silver chloride disk electrodes placed on the forehead (+ve), and on the mastoid ipsilateral to the stimulated ear (-ve). A similar electrode was placed on the contralateral mastoid to act as a ground. Interelectrode impedance was less than $10 \mathrm{kohms}$ at 260 Hz. The EEG signal was amplified by a custom built preamplifier and filtered using a band pass of 0.2 to $10 \mathrm{kHz}$.

Stimulus generation and waveform analysis were as described by Cohen et al. (1991). Fourier analyses of the EEG signal were performed at the fundamental and second harmonic of the modulation frequency using analog multiplication followed by lowpass filtering (Regan, 1966). For each harmonic, two multipliers and two low-pass filters were used to extract both phase and amplitude information. An IBM-compatible XT-type microcomputer sampled the output of the four low-pass filters every 32 modulation periods. Only the fundamental fre- 
quency of the modulation frequency was used in determining the presence of a response.

Within the system, a response was rejected if its noise level exceeded $0.5 \mu V p-p$. This value was determined as described in Cohen et al. (1991).

The presence or absence of a response was determined automatically using a detection criterion which looked for nonrandom phase behavior. This was equivalent to the phase coherence technique described by Jerger et al. (1986) and Stapells et al. (1987). The system monitored a function that calculated the probability that the set of response angles, at any given time during the run, could have arisen in the absence of a response component at the modulation frequency. The essential criterion for a response was that the probability function be less than 0.01 . However, because the probability function was monitored continuously throughout a run, a distortion of the statistics occurred which resulted in an increased probability of a false response. A response was accepted, therefore, if that probability function remained less than 0.01 for a time equal to one-tenth of the run time that elapsed when it was first found to be less than 0.01 . This criterion resulted in a false response probability of $p<0.03$ (for detection of the response component at the fundamental of the modulation frequency). The run was terminated when the detection criterion was satisfied or when the maximum allowed time had elapsed.

The time taken to detect a response was generally in the range of 20 to $90 \mathrm{sec}$, depending on the signal to noise ratio of the SSEP response and the electroencephalographic noise. Overall, the testing of each subject took approximately 30 to 60 minutes.

Stimuli were 250-, 500-, 1000-, 2000-, and $4000-\mathrm{Hz}$ tones amplitude and frequency modulated at a rate of $90 \mathrm{~Hz}$. A modulation frequency of $90 \mathrm{~Hz}$ was selected to ensure that responses with latencies of about 10 msec were evoked at each carrier frequency. An amplitude modulation depth of $100 \%$, and a frequency modulation width of $10 \%$ were combined to maximize the amplitude of the evoked response (Cohen et al., 1991). Calibration levels for pure tones were provisional levels provided by the manufacturer (Etymotic Research, 1985), and were acquired using a Phonic Ear HA-1 (2 cc) coupler, a B \& K condenser microphone, a B \& K 2613 amplifier, and a B \& K 2120 frequency analyzer. The tones were presented via Etymotic Research ER-3A tubephones with foam EAR plugs or mu-metal shielded TDH-39 headphones. These headphones allowed maximum sound levels of $104 \mathrm{~dB}$ HL for the $250 \mathrm{~Hz}$ carrier, and $120 \mathrm{~dB}$ HL for the 500-, 1000-, 2000-, and $4000-\mathrm{Hz}$ carrier frequencies.

To obtain SSEP thresholds the level of the stim- ulus was decreased in $10 \mathrm{~dB}$ steps until no response could be detected. It was then increased in 5 -dB steps until the response could again be identified. Threshold was defined as the minimum level at which the response could be automatically detected by the system. Thresholds were determined in this way for each of the subjects using stimuli with a range of carrier frequencies presented to each ear separately. It should be noted that for some subjects, particularly the children in natural sleep, clinical constraints (such as the subject waking up before the expected time) prevented the acquisition of thresholds at all frequencies.

\section{Results}

\section{Section 1: The Relationship Between SSEP and Behavioral Threshold}

Figure 1 shows the SSEP and behavioral thresholds obtained at frequencies of 250-, 500- 1000-, $2000-$, and $4000 \mathrm{~Hz}$ for three subjects with varying degrees of sensorineural hearing loss. The relationship between SSEP and behavioral levels seen in these audiograms is typical of those observed in the subjects that we tested, with the evoked potential thresholds more closely following the pattern of the hearing levels as they increase.

The absence of SSEP responses at 2000 and 4000 $\mathrm{Hz}$ in the third of these examples is also typical of the results obtained in subjects at frequencies at which no threshold was observed behaviorally. This finding confirms that at high stimulus levels, artifactual responses were not contaminating the results.

Four hundred thirteen comparisons of behavioral and SSEP thresholds have been made. In instances where behavioral or SSEP responses were absent, these comparisons were not included in the final data analysis. In 73 instances, both behavioral and SSEP responses were absent at the audiometric limits. In 13 instances, behavioral responses were present, but SSEP responses were absent. In eight instances, SSEP responses were present, but behavioral responses were absent. In these 21 cases where either behavioral or SSEP responses were absent, (but not both), the responses that were measured were still within $10 \mathrm{~dB}$ of the absent response. Thus, the elimination of these absent cases from the final data analysis was not considered to have a significant effect on the overall findings.

The relationship between behavioral and SSEP thresholds from both ears for all of the subjects is shown in Figure 2. The data have been fitted with linear regression lines. The regression lines, with their slopes of less than unity, reflect that the SSEP threshold is closer to behavioral threshold in ears 

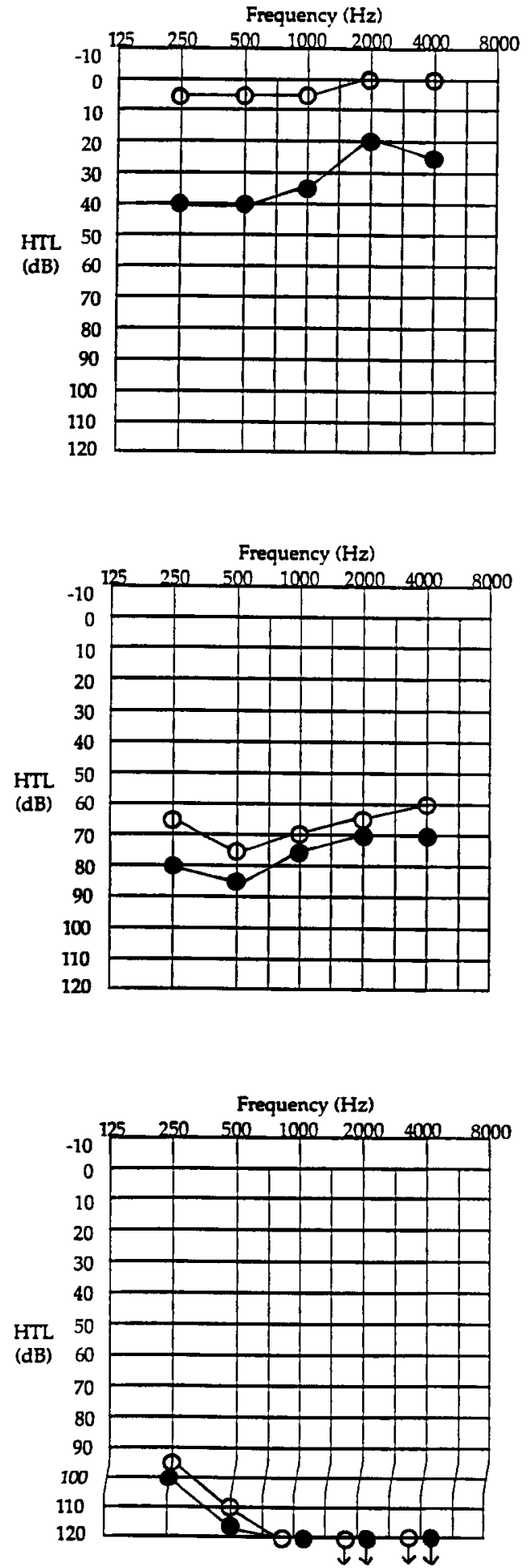

Figure 1. The behavioral and SSEP audiograms for three subjects with varying degrees of hearing acuity. Open circles represent behavioral pure tone thresholds; filled circles show the SSEP thresholds obtained with an AM/FM modulated stimulus. A modulation frequency of $90 \mathrm{~Hz}$ was used.

with a greater degree of hearing loss. The lower $\mathrm{Y}$ intercepts and higher regression line gradients seen with increases in carrier frequency also indicate that more SSEP thresholds are closer to behavioral threshold with high frequency stimuli.

The relationship between SSEP and behavioral thresholds is demonstrated further by examining the correlation coefficient calculated at each of the carrier frequencies. As seen in Table 1, the correlation coefficients are all greater than 0.95 . An overall correlation coefficient of 0.97 for all of the frequencies was obtained. Thus, a very strong positive relationship exists between the behavioral and SSEP threshold.

Table 2 lists the standard deviation values for the SSEP thresholds about the regression lines at each carrier frequency for the three hearing loss categories. The categories were selected to provide similar numbers of data points in each group. The categories were based on the subjects' behavioral thresholds and were as follows:

category 1: 0-35 dB HL; category 2: $40-75 \mathrm{~dB} \mathrm{HL}$; category $3: 80+\mathrm{dB}$ HL.

This table shows that the variability expressed as a standard deviation of the SSEP threshold decreases with increasing frequency and increasing degree of the loss. An $F$-ratio was used to examine differences in variability across frequencies and across hearing loss categories. Overall, the spread of low-frequency SSEP thresholds was greater than the spread of high-frequency SSEP thresholds. Specifically, $250 \mathrm{~Hz}$ was significantly more variable than $2000 \mathrm{~Hz}, F(75,85)=2.033, p<0.01$, and 4000 $\mathrm{Hz}, F(75,77)=2.532, p<0.001$, but not more variable than $500 \mathrm{~Hz}$ or $1000 \mathrm{~Hz} ; 500 \mathrm{~Hz}$ was significantly more variable than $2000 \mathrm{~Hz}, F(88,83)$ $=2.056, p<0.001$ and $4000 \mathrm{~Hz}, F(88,77)=2.559$, $p<0.001$, but not more variable than $1000 \mathrm{~Hz} ; 1000$ $\mathrm{Hz}$ was significantly more variable than $2000 \mathrm{~Hz}$, $(85,83)=1.763, p<0.01$, and $4000 \mathrm{~Hz} F(85,77)=$ $2.195, p<0.001$. There was no significant difference between $2000 \mathrm{~Hz}$ and $4000 \mathrm{~Hz}$. Further, the standard deviations for milder losses were greater than for more severe losses. Specifically, hearing loss category one was significantly more variable than category two $F(134,139)=2.882, p<0.001$, and category three $F(134,137)=2.392, p<0.001$. The difference between categories two and three was not significant.

\section{Section 2: Prediction of Behavioral Threshold}

The previous section has shown that a very strong relationship between SSEP and behavioral threshold exists. Further, the amount of variance about each regression line does not appear to be large. In fact, the data points lie within $10 \mathrm{~dB}$ of the regression lines on 390 of the $413(94 \%)$ occasions. This lack of variability suggests that the regression lines 


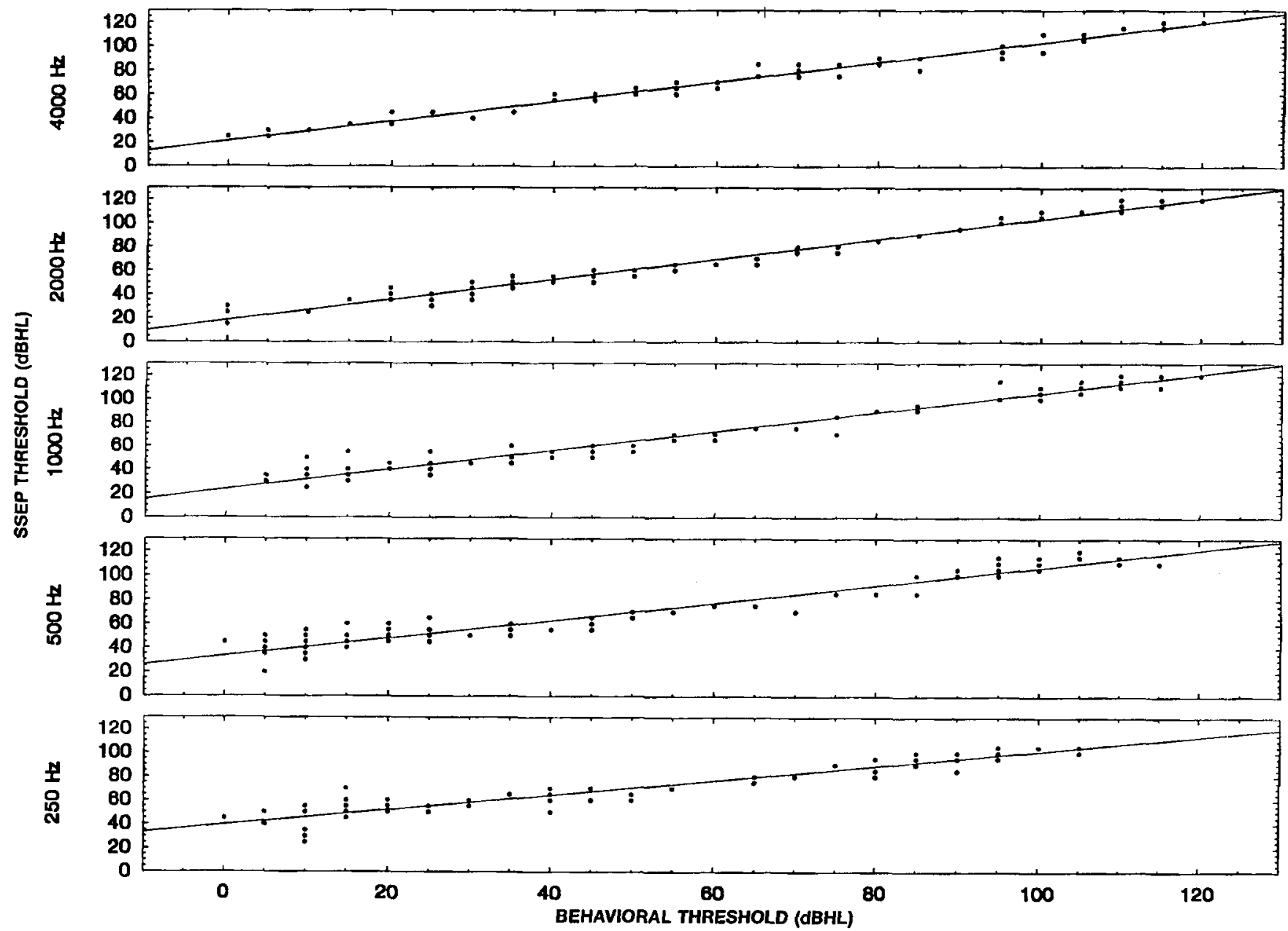

Figure 2. The relationship between behavioral and SSEP thresholds to 250-, 500-, 1000-, 2000-, and 4000-Hz tones amplitude and frequency modulated at a rate of $90 \mathrm{~Hz}$. Shown are the results for 60 subjects with varying degrees of sensorineural hearing loss.

could be used to predict behavioral thresholds from recorded SSEPs.

Although Figure 2 shows the conventional way of

TABLE 1. Pearson product-moment correlation coefficient comparing SSEP and behavioral thresholds for each of the carrier frequencies.

\begin{tabular}{llllll}
\hline & \multicolumn{5}{c}{ Frequency $(\mathrm{Hz})$} \\
\cline { 2 - 6 } & 250 & 500 & 1000 & 2000 & 4000 \\
\hline Pearson $r$ & 0.96 & 0.97 & 0.98 & 0.99 & 0.99 \\
\hline
\end{tabular}

TABLE 2. Standard deviation values $(\mathrm{dB})$ illustrating the spread of SSEP thresholds about the lines of best fit for each carrier frequency and each of the hearing loss categories.

\begin{tabular}{lccccc}
\hline \multicolumn{1}{c}{ Category } & $250 \mathrm{~Hz}$ & $500 \mathrm{~Hz}$ & $1 \mathrm{kHz}$ & $2 \mathrm{kHz}$ & $4 \mathrm{kHz}$ \\
\hline $1(0-35 \mathrm{~dB} \mathrm{HL})$ & 8.2 & 7.6 & 7.3 & 5.7 & 4.2 \\
$2(40-75 \mathrm{~dB} \mathrm{HL})$ & 5.0 & 3.4 & 3.8 & 3.3 & 3.9 \\
3 (80 + dB HL) & 5.0 & 5.3 & 4.5 & 3.2 & 4.4 \\
Frequency total & 6.5 & 6.5 & 6.1 & 4.6 & 4.1 \\
\hline
\end{tabular}

representing SSEP and behavioral thresholds, the clinical task usually involves predicting behavioral thresholds from SSEP levels. Consequently, the axes in Figure 2 have been reversed so as a fairer representation of the amount of variance about each regression line can be seen. Figure 3 demonstrates this relationship between behavioral and SSEP thresholds. It can be seen that the amount of variance about each regression line is still comparatively small.

The data in Figure 3 have been used to create a set of formulae that could be used to predict behavioral thresholds from SSEP thresholds. These formulae are dependent on the testing paradigm used in this study. The formulae at each of the carrier frequencies are as follows:

$$
\begin{aligned}
& \mathrm{y}(\mathrm{x})=1.477 \mathrm{x}-53.469(250 \mathrm{~Hz}) \\
& \mathrm{y}(\mathrm{x})=1.295 \mathrm{x}-40.197(500 \mathrm{~Hz}) \\
& \mathrm{y}(\mathrm{x})=1.182 \mathrm{x}-26.070(1000 \mathrm{~Hz}) \\
& \mathrm{y}(\mathrm{x})=1.045 \mathrm{x}-19.463(2000 \mathrm{~Hz}) \\
& \mathrm{y}(\mathrm{x})=1.193 \mathrm{x}-23.581(4000 \mathrm{~Hz})
\end{aligned}
$$



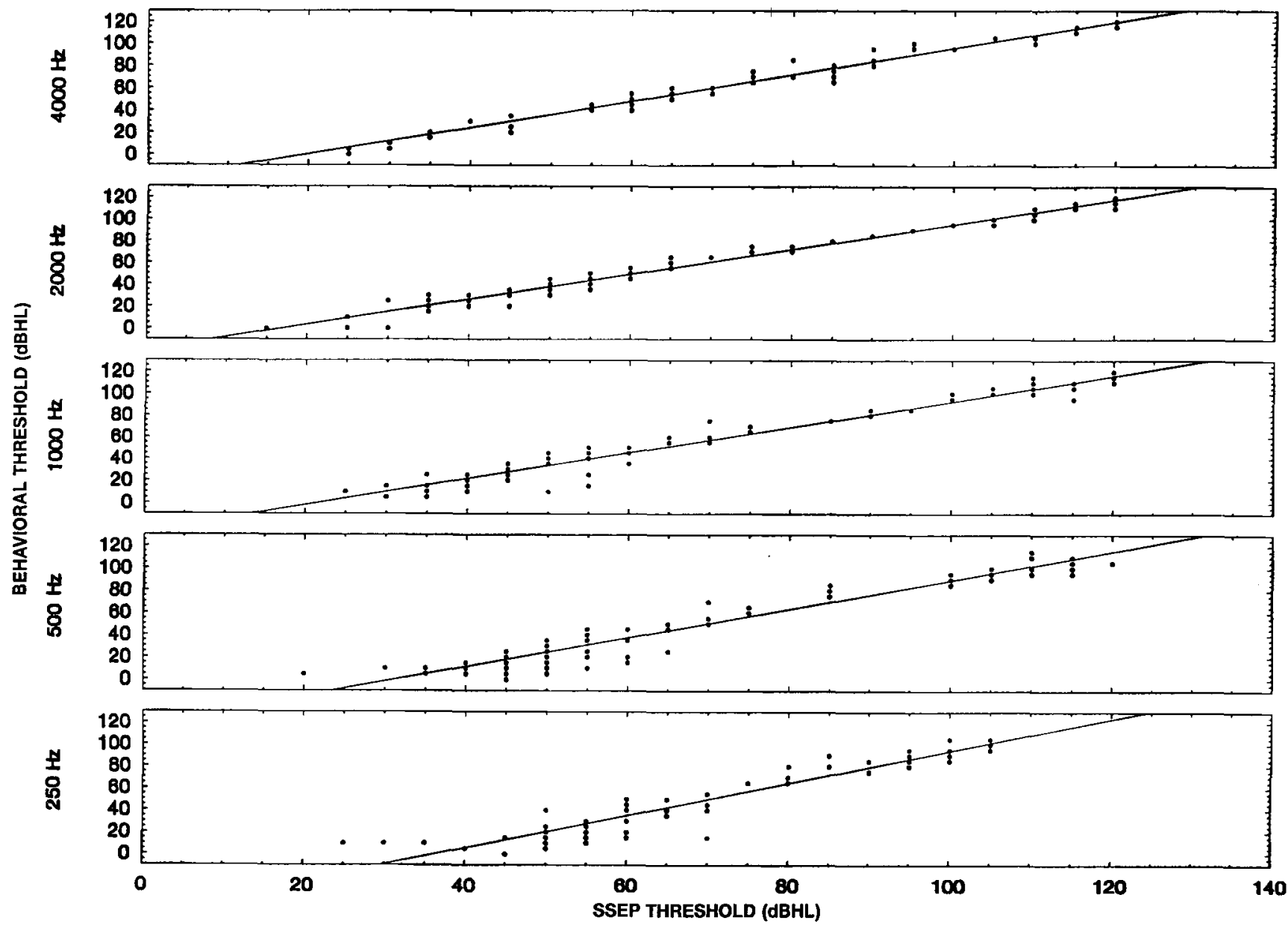

Figure 3. Regression lines fitted to behavioral versus SSEP thresholds for all subjects for the frequencies 250-, 500-, 1000-, 2000-, and $4000-\mathrm{Hz}$, with behavioral thresholds on the dependent variable.

where: $\mathrm{y}=$ behavioral threshold (predicted); $\times=$ SSEP threshold.

Behavioral prediction errors were generated for each of the carrier frequencies based on the linear regression data in Figure 3. These errors were obtained by subtracting the predicted behavioral threshold from the actual behavioral threshold.

A two-way analysis of variance comparing the mean behavioral prediction errors for the adult and child groups showed no significant difference or interaction effect between the two age groups at any of the carrier frequencies.

The standard deviations of the prediction errors for each carrier frequency and for each of the three hearing loss categories are listed in Table 3 . The threshold criteria for determining hearing loss categories were selected to provide similar numbers of data points in each group. The categories were based on the obtained SSEP thresholds and were as follows:

category 1: 0-55 dB HL; category 2: $60-85 \mathrm{~dB}$ HL; category 3: $90+\mathrm{dB} H \mathrm{HL}$.

This table shows that the error in the prediction of behavioral threshold decreases with increasing hearing loss and with increasing carrier frequency. Overall, the low frequency errors were more variable than the high frequency errors. Specifically, $250 \mathrm{~Hz}$ was more variable than $1000 \mathrm{~Hz}, F(75,85)=$ $1.896, p<0.01$, and $2000 \mathrm{~Hz}, F(75,83)=3.614, p<$ 0.001 , and $4000 \mathrm{~Hz}, F(75,77)=4.140, p<0.001$, but not more variable than $500 \mathrm{~Hz} ; 500 \mathrm{~Hz}$ was more variable than $2000 \mathrm{~Hz}, F(88,83)=2.710, p<0.001$, and $4000 \mathrm{~Hz}, \mathrm{~F}(88,77)=3.105 p<0.001$, but not more variable than $1000 \mathrm{~Hz} ; 1000 \mathrm{~Hz}$ was more variable than $2000 \mathrm{~Hz}, F(85,83)=1.906 p<0.01$,

TABLE 3. Standard deviation values (dB) of the errors of the predicted behavioral threshold from the SSEP threshold and the actual behavioral threshold for five carrier frequencies and three categories of hearing loss.

\begin{tabular}{lccccc}
\hline \multicolumn{1}{c}{ Category } & $250 \mathrm{~Hz}$ & $500 \mathrm{~Hz}$ & $1 \mathrm{kHz}$ & $2 \mathrm{kHz}$ & $4 \mathrm{kHz}$ \\
\hline $1(0-55 \mathrm{~dB} \mathrm{HL})$ & 11.9 & 9.6 & 8.6 & 6.3 & 4.7 \\
$2(60-85 \mathrm{~dB} \mathrm{HL})$ & 11.6 & 9.6 & 5.7 & 3.9 & 5.1 \\
$3(90+\mathrm{dB} \mathrm{HL})$ & 6.5 & 6.2 & 5.4 & 3.6 & 4.8 \\
Total & 10.1 & 8.7 & 7.3 & 5.3 & 4.9 \\
\hline
\end{tabular}


and $4000 \mathrm{~Hz} F(85,77)=2.183 p<0.001$. There was no significant difference between $2000 \mathrm{~Hz}$ and 4000 $\mathrm{Hz}$. Further, the errors were more variable for the milder losses than for the more severe losses. Significant differences in the spread of behavioral thresholds were observed between hearing loss category one and category three, $F(147,125)=2.518, p$ $<0.001$ and categories two and three, $F(138,125)=$ $1.952, p<0.001$. The difference between categories one and two was not significant.

\section{Discussion}

The results presented in this paper indicate that linear regression analyses can be used to describe the relationship between behavioral and steadystate evoked potential thresholds obtained in sleeping subjects when using a modulation rate of $90 \mathrm{~Hz}$. Furthermore, the data has shown that for each of the carrier frequencies ( 250 to $4000 \mathrm{~Hz}$ ), estimates of behavioral levels can be made from SSEP thresholds with reasonable confidence.

Two aspects of the SSEP/behavioral threshold relationship are illustrated by the scatter plots presented in Figure 2. First, the data indicate that SSEP thresholds obtained from hearing-impaired subjects more closely match behavioral thresholds as the degree of hearing loss increases. This may be partly explained by the possible presence of recruitment resulting in a more pronounced transition from below threshold nonresponse, to above threshold response in ears with sensorineural losses. For the normally hearing subjects in this study, the SSEP thresholds were approximately $17 \mathrm{~dB} \mathrm{HL}$ $(4000 \mathrm{~Hz})$ to $35 \mathrm{~dB} \mathrm{HL}(250 \mathrm{~Hz})$ greater than the behavioral thresholds. This elevation in SSEP thresholds has also been obtained in other evoked potential studies using normal hearing subjects. For example, the Gorga et al. (1988) study of ABR thresholds to tones in quiet found similar levels of evoked potential threshold elevation with their normally hearing subjects.

A second aspect of the SSEP/behavioral threshold relationship seen in the scatter plots is that the regression lines fitted to the higher frequency data have higher gradients and lower $Y$ intercepts than the regression lines fitted to the lower frequencies. This illustrates that SSEP thresholds are closer to behavioral threshold when higher carrier frequency stimuli are used. This is consistent with the findings of Cohen et al. (1991) that larger SSEP response amplitudes are observed in sleeping subjects when high frequency stimuli are employed. The poorer sensitivity at low frequencies is probability best explained, as with transient responses by the notion of poorer neural synchronization.
Given the lower response amplitudes of SSEPs in the lower frequencies (Cohen et al., 1991), and the poorer sensitivity of low frequency SSEPs in the present study, it is possible that some of the observed SSEP thresholds could have originated from regions of the cochlea more basal than those associated with the nominal frequencies of the stimuli. However, the way in which the SSEP thresholds mirror the behavioral levels in the audiograms presented in Figure 1, does suggest that appropriate regions of the basilar membrane are being stimulated. Further, the sensitivity of the SSEP thresholds in the low frequencies appears to be independent of the degree of the behavioral hearing loss in the high frequencies. Evidence for the frequency specificity of the response can be seen in the third of these audiograms where the SSEP threshold levels (SPL) at $250 \mathrm{~Hz}$ and $500 \mathrm{~Hz}$ are in fact lower than the behavioral levels in the octave frequencies above.

The clinical relevance of the SSEP procedure or any other objective measure of hearing lies in its ability to reliably predict behavioral levels. Although estimates of the average behavioral threshold corresponding to a particular SSEP threshold can be calculated from the linear regression line, the degree of confidence with which such predictions can be made is determined by the spread of likely thresholds about that regression line.

Behavioral threshold prediction errors derived from our data indicated that the range of behavioral levels which could correspond to a particular SSEP threshold was in fact, reasonably small. The prediction errors also varied with carrier frequency, and were smallest for the higher frequencies. Standard deviations calculated for the low frequency stimuli, did however also show a low level of variability, particularly in ears with severe to profound hearing losses. Thus, when using regression lines to predict behavioral thresholds from SSEP thresholds, one can assume that the predicted threshold will be close to the actual threshold at all of the octave frequencies tested.

A number of advantages of our SSEP technique over other evoked potential techniques were identified in this study. First, the variability of the SSEP thresholds presented in this paper compare favorably with other objective, frequency specific methods of hearing estimation. For example, in an ABR study by Stapells et al. (1990) employing short duration tones in notched noise, overall standard deviations describing the spread of evoked thresholds about their regression lines appear to be larger than those shown in our study. It should be noted that there were fewer normally hearing subjects in our study compared with Stapells et al., and as we have 
demonstrated, results become less variable as degree of hearing loss increases. However, the standard deviations derived from our best hearing ears ( 0 to $35 \mathrm{~dB} \mathrm{HL}$ ), were smaller than the overall values presented by Stapells et al., and with the exception of the $500 \mathrm{~Hz}$ data, were smaller than those obtained from even their hearing impaired group.

In a further study investigating ABR thresholds elicited in response to tone bursts in quiet (Gorga et al., 1988), ABR thresholds were more variable than the SSEP thresholds obtained in this study particularly at the lower frequencies. Gorga et al. acknowledged that this greater variability in the lower frequencies limited the clinical application of predicting behavioral thresholds from ABR thresholds, as corrections to tone burst normative ABR data in the low frequencies cannot be formulated. In contrast, the smaller variability in SSEP thresholds in our study may enable more reliable predictions of behavioral threshold to be made even at the lower frequencies.

A second advantage of the SSEP technique used in this study is the automatic nature of the response detection system. The periodicity of the steady-state evoked potential allowed the presence or absence of a response to be easily established automatically using Fourier and statistical analysis techniques. The error associated with subjective waveform interpretation was therefore avoided. An automatic detection technique is particularly useful when trying to detect responses at low levels of the stimulus as response amplitudes tend to be smaller than at the higher levels. As the SSEP technique reduces the need for subjective evaluation of the response, values close to or at threshold can thus be more easily obtained. Furthermore, the automatic detection system may in part explain the small variability in SSEP thresholds discussed previously.

A third advantage that the SSEP procedure has over transient evoked response techniques is the ability to record responses for greater degrees of hearing loss. The continuously modulated tone used to elicit the SSEP response can be presented at levels as high as $120 \mathrm{~dB}$ HL. In contrast, click and brief tone procedures are typically restricted to presentation levels up to $100 \mathrm{~dB}$ nHL. Click and brief tone procedures are usually insufficient for assessment of profound hearing losses. In contrast, the results obtained from the 18 profoundly deaf subjects in this study indicated that even at high stimulus levels, SSEP assessments could provide accurate estimates of hearing across the frequency range.

A further advantage of the SSEP technique is the speed in which a response can be detected. In most cases, a response could be observed within 20-90 s after the onset of the stimulus. In fact, many responses were clearly detected within the first $30 \mathrm{~s}$. Thus, the response detection system used in this study is very fast, and hence reduces the need to have the subject asleep or under sedation for long periods of time. As a result, the clinician is more likely to obtain all the information that is required before the subject awakens, and within one testing period.

The findings of this study also showed that there was no significant age effect in error prediction values at each of the frequencies tested. Consequently, the SSEP technique can be used with equal confidence when assessing the hearing status of adults or children, making it highly applicable to a variety of test situations. In particular, unlike some other evoked potential procedures, the SSEP technique is well suited to the assessment of hearing in young children. Where middle latency and slow cortical potentials for example, have been shown to be unreliable in this group as a result of both maturational and sleeping effects (Kraus, Smith, Reed, \& Stein, 1985; Picton \& Durieux-Smith, 1978; Stapells, Galambos, \& Costello, 1988), the SSEP response when elicited by stimuli modulated at a sufficiently high rate, has been consistently recordable in neonates (Rickards et al., 1994). Furthermore, the prediction errors in this study when compared across response state showed no significant differences between subjects in natural sleep, under the sedative chloral hydrate, or under a general anesthetic. This result is consistent with other studies which have suggested that transient evoked potentials with similarly short latencies (such as the ABR) have been unaffected by sedatives and anesthetics (Duncan, Sanders, \& McCullough, 1979). Although Cohen et al. (1991) have shown that the amplitude of the response is dependent on the various sleep stages, the results of the present study have shown that overall, sleep does not significantly affect the relationship between SSEP and behavioral thresholds. This finding further demonstrates the utility of the $90 \mathrm{~Hz}$ SSEP procedure in the assessment of uncooperative subjects.

In conclusion, this study suggests that SSEP thresholds recorded in sleeping subjects can be used to make frequency specific estimates of hearing thresholds. Auditory threshold estimation is important in the identification of hearing loss in young children. Early diagnosis, and the subsequent audiological management including hearing aid fitting is vital for children with significant hearing impairments. The data presented in this study suggest that very young children can be accurately assessed using SSEPs. 
Address for correspondence: Professor Field W. Rickards, Deafness Studies Unit, Department of Learning, Assessment and Special Education, University of Melbourne, Parkville VIC. 3052 Australia.

Received October 3, 1994; accepted April 26, 1995

\section{REFERENCES}

Aoyagi, M., Kiren, T., Kim, Y., Suzuki, Y., Fuse, T., \& Koike, Y. (1993). Optimal modulation frequency for amplitude-modulation following response in young children during sleep. Hearing Research, 65, 253-261.

Beattie, R. C., \& Kennedy, K. M. (1992). Auditory brainstem response to tone bursts in quiet, notch noise, highpass noise, and broadband noise. Journal of the American Academy of Audiology, 3, 349-360.

Brown, D., \& Shallop, J. (1982). A clinically useful $500 \mathrm{~Hz}$ evoked response. Nicolet Potentials, 1, 9-12.

Cohen, L. T., Rickards, F. W., \& Clark, G. M. (1991). A comparison of steady-state evoked potentials to modulated tones in awake and sleeping humans. Acoustical Society of America, 90, 2467-2479.

Duncan, P. G., Sanders, R. A., \& McCullough, D. W. (1979). Preservation of auditory-evoked brainstem responses in anaesthetised children. Canadian Anaesthesiology Society Journal, $26,492-495$.

Etymotic Research. (1985). Etymotic research ER-3a tubephone insert earphone calibration instructions. Etymotic Research. 61 Martin Lane, Elk Grove Village, IL 60007.

Galambos, R., Makieg, S., \& Talmachoff, P. J. (1981). A $40 \mathrm{~Hz}$ auditory potential recorded from the human scalp. Proceedings of the National Academy of Sciences of the united States of America, 78, 2643-2647.

Gorga, M. P, Kaminski, J. R., Beauchaine, K. A., \& Jesteadt, W. (1988). Auditory brainstem responses to tone bursts in normally hearing subjects. Journal of Speech and Hearing Research, 31, 87-97.

Hawes, M. D., \& Greenberg, H. J. (1981). Slow brainstem responses (SN 10) to tone pips in normally hearing newborns and adults. Audiology, 20, 113-122.

Jerger, J., Chimiel, R., Frost, J. D. Jr., \& Coker, N. (1986). Effect of sleep on the auditory steady-state evoked potential. Ear and Hearing, 7, 240-245.

Kraus, N., Smith, D. I., Reed, N. L., \& Stein, L. K. (1985). Auditory middle latency responses in children: Effects of age and diagnostic category. Electroencephalography and Clinical Neurophysiology, 62, 343-351.

Kuwada, S., Batra, R., \& Maher, V. L. (1986). Scalp potentials of normal and hearing impaired subjects in response to sinusoidally amplitude-modulated tones. Hearing Research, 21, 179192.

Levi, E. C., Folsom, R. C., \& Dobie, R. A. (1993). Amplitudemodulation following response (AMFR): Effects of modulation rate, carrier frequency, age and state. Hearing Research, 5, $366-370$.
Markides, A. (1986). Age of fitting of hearing aids and speech intelligibility. British Journal of Audiology, 20, 165-168.

Osterhammel, P. A., Shallop, J. K., \& Terkildsen, K. (1985). The effect of sleep on the auditory brainstem response (ABR) and the middle latency response (MLR). Scandinavian Audiology, $14,47-50$.

Picton, T. W., \& Durieux-Smith, A. (1978). The practice of evoked potential audiometry. Otolaryngology Clinics of North America, 11, 263-282.

Picton, T. W., Durieux-Smith, A., \& Moran, L. (1994). Recording auditory brainstem responses from infants. International Journal of Paediatric Otorhinolaryngology, 28, 93-110.

Picton, T. W., Vajsra, J., Rodriguez, R., \& Campbell, K. B. (1987), Reliability estimates from steady-state evoked potentials. Electroencephalography and Clinical Neurophysiology, 68, 119131.

Ramkalawan, T. W., \& Davis, A. C. (1992). The effects of hearing loss and age at intervention on some language metrics in young hearing impaired children. British Journal of Audiology, 26, 97-107.

Regan, D. (1966). Some characteristics of average steady-state and transient responses evoked by modulated light. Electroencephalography and Clinical Neurophysiology, 20, 238-248.

Rickards, F. W., \& Clark, G. M. (1984). Steady-state evoked potentials to amplitude modulated tones. In Evoked Potentials II (pp. 163-168). Boston: Butterworth.

Rickards, F. W., Tan, L. E., Cohen, L. T., Wilson, O. J. Drew, J. H., \& Clark, G. M. (1994). Auditory steady-state evoked potentials in newborns. British Journal of Audiology, 28, 327-337.

Shallop, J. K. (1983). Electric response audiometry: the morphology of normal responses. Advances in Oto-Rhino-Laryngology, 29, 124-139.

Shallop, J. K., \& Osterhammel, P. A. (1983). A comparative study of measurements of SN 10 and the $40 / \mathrm{sec}$ middle latency responses in newborns. Scandinavian Audiology, 12, 91-95.

Stapells, D. R. (1989). Auditory brainstem response assessment of infants and children. Seminars in Hearing, 10, 229-251.

Stapells, D. R., Galambos, R., \& Costello, J. (1988). Inconsistency of auditory middle latency response in infants. Electroencephalography and Clinical Neurophysiology, 71, 289-295.

Stapells, D. R., Makieg, G., \& Galambos, R. (1987). Auditory steady-state response threshold prediction using phase coherence. Electroencephalography and Clinical Neurophysiology, 67, 260-270.

Stapells, D. R., Picton, D. W., Durieux-Smith, A., Edwards, C. G., \& Moran, L. M. (1990). Thresholds for short latency auditoryevoked potentials to tones in notched noise in normal hearing and hearing impaired subjects. Audiology, 29, 262-274.

\section{Reference Note}

1 Stapells, D. R., Picton, D. W., \& Durieux-Smith, A. (1983). Prediction of audiometric thresholds in normal and hearingimpaired subjects using the $40 \mathrm{~Hz}$ event related potential. Canadian Speech and Hearing Association annual convention, 13 May 1983, Montreal, Canada. 


\section{University Library}

\section{- M M I N E R VA A gateway to Melbourne's research publications}

Minerva Access is the Institutional Repository of The University of Melbourne

Author/s:

RANCE, GARY;Rickards, Field W.;Cohen, Lawrence T.;De Vidi, Sandra;Clark, Graeme M.

Title:

The automated prediction of hearing thresholds in sleeping subjects using auditory steadystate evoked potentials

Date:

1995

\section{Citation:}

Rance, G., Rickards, F. W., Cohen, L. T., De Vidi, S., \& Clark, G. M. (1995). The automated prediction of hearing thresholds in sleeping subjects using auditory steady-state evoked potentials. Ear and Hearing, October, 16(5), 499-507.

Persistent Link:

http://hdl.handle.net/11343/27453 